\title{
Worst Imaginable Health
}

National Cancer Institute

\section{Source}

National Cancer Institute. Worst Imaginable Health. NCI Thesaurus. Code C111679.

The worst state of well-being an individual can conceive of experiencing. 